\title{
Tumor pulmonar con metástasis prostática (a propósito de un caso)
}

\section{Lung Cancer metastasizing to the prostate: Case report and literature review}

\section{Sr. Director:}

La glándula prostática muy rara vez es asiento de tumores metastásicos. Incluso en textos urológicos y anatomopatológicos es difícil encontrar clasificaciones que incluyan las neoplasias secundarias prostáticas. La incidencia de tumores secundarios prostáticos varía de unas series a otras debido a la disparidad de los criterios de inclusión de casos, de la población estudiada y de los tipos tumorales analizados ${ }^{1}$.

El motivo de este artículo es revisar la bibliografía y poner en relevancia la rareza de la afectación metastásica del aparato genitourinario, en especial de la próstata.

Presentamos el caso de un paciente de 82 años diagnosticado de carcinoma no microcítico de pulmón en lóbulo superior izquierdo (Tumor de Pancoast), (fig. 1). Recibió quimioterapia con Taxol-Cisplatino y radioterapia sobre la tumoración y posteriormente se completó el tratamiento mediante lobectomía y braquiterapia de alta tasa perioperatoria. Pasados 4 años presenta recurrencia y se realiza nueva lobectomía con resultado anatomopatológico de carcinoma mixto (adenocarcinoma y epidermoide) T3, N2. Acude de nuevo a consulta para recibir valoración urológica 5 años después del primer diagnóstico, por infección del tracto urinario refractaria a tratamiento antibiótico. En el momento de la consulta se encontraba libre de enfermedad. Previamente el paciente

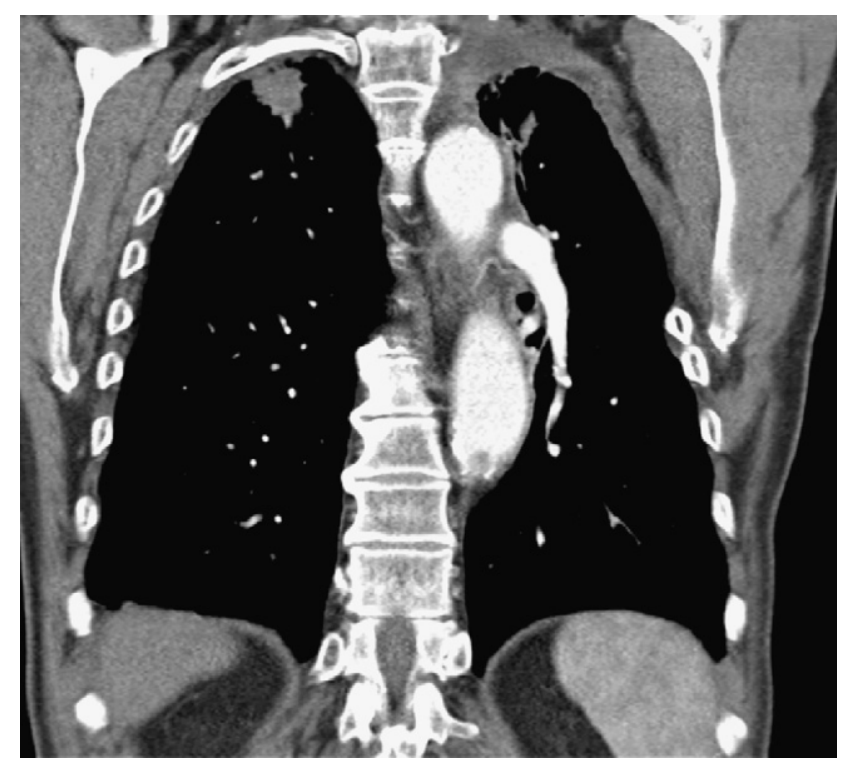

Figura 1 - Imágen de TAC en la que se aprecia tumor pulmonar en lóbulo superior izquierdo (Tumor de Pancoast). refería una disminución del caudal miccional y disuria de meses de evolución. El PSA realizado en el momento de la consulta fue de $4,4 \mathrm{ng} / \mathrm{ml}$. El tacto rectal y la ecografía no fueron relevantes. Se le diagnostica de hiperplasia benigna de próstata e infección del tracto urinario complicada pautándole nuevo tratamiento antibiótico y alfabloqueantes. Una semana después presentó cuadro de retención aguda de orina y finalmente se le indicó una resección transuretral prostática. El informe anatomopatológico reveló una afectación metastásica con extensa invasión linfática y vascular del parénquima prostático, por un carcinoma pobremente diferenciado de origen pulmonar. (fig. 2). Las células metastásicas mostraron una fuerte

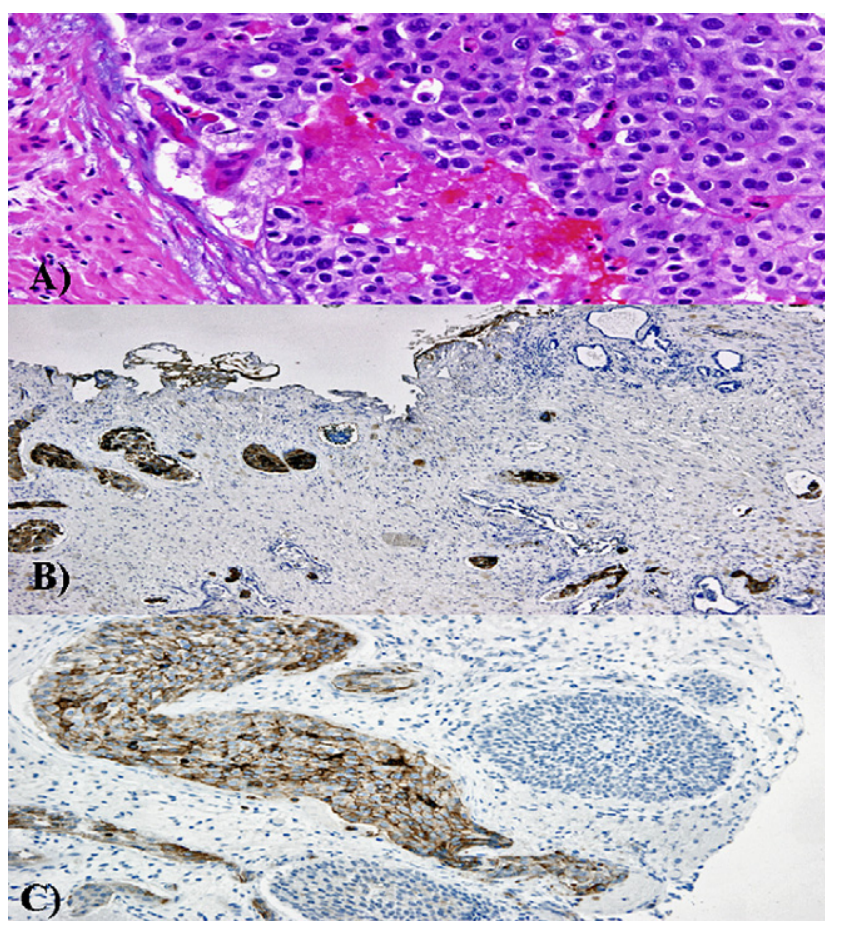

Figura 2 - A) H\&E ( × 300). Se aprecian los focos metastásicos del tumor de células escamosas pobremente diferenciado entre glándulas prostáticas normales. B) Citokeratina-7 IHC ( $\times 100)$. Las células metastásicas mostraron una fuerte y difusa inmunopostividad citoplamática para la CK-7. Las glándulas prostáticas no presentaron la citada inmunorreactividad. Las células tumorales presentaron negatividad para PSA, p63, y uroplakina-III. C) Mesotelina IHC ( $\times 100)$. Las células metastásicas mostraron una fuerte y difusa inmunopostividad de membrana para la mesotelina, siendo negativa en el caso de las células uroteliales. 
inmunopostividad citoplamática de tipo difuso para la CK-7 e inmunorreactividad de membrana para la mesotelina. Además presentaron negatividad para PSA, p63, y uroplakina-III. El TAC torácico que se le realizó posteriormente no pudo concluir existencia de recidiva pulmonar ni ganglionar. Se remitió el caso al servicio de oncología iniciándose un primer ciclo de quimioterapia con Pemetrexed y Carboplatino. Al mes ingresa por cuadro disneico realizándose nuevo TAC Torácico en el que se evidencia progresión pulmonar falleciendo a las dos semanas por un episodio de insuficiencia respiratoria aguda.

Una abrumadora mayoría de los cánceres de próstata (90-95\%) son adenocarcinomas típicos ${ }^{2}$. El 5-10\% restante corresponde a formas poco frecuentes de adenocarcinomas, sarcomas o tumores de células transicionales prostáticos sin afectación vesical. Las neoplasias secundarias de próstata también pertenecen a este grupo y suponen el 2,1\% de todas las neoplasias sólidas de afectación prostática en especímenes quirúrgicos ${ }^{1}$. Otras series hablan de un 0,5\%-5,6\% de tumores secundarios próstaticos en pacientes que mueren por neoplasia ${ }^{3,4}$, y del $0,01 \%$ en pacientes sometidos a tratamiento por cáncer de próstata.

El subtipo histológico más frecuente de tumor sólido secundario prostático es el de células transicionales (57\% de los casos). Su origen es traducción de la invasión directa de la próstata por tumores vesicales de células transicionales ${ }^{6}$. El adenocarcinoma sería el segundo en frecuencia con un $20 \%$ de los casos y los siguientes el carcinoma de células pequeñas (8\%) y el melanoma $4 \%^{7}$.

La afectación del tracto genitourinario (incluyendo la próstata) por neoplasias secundarias se puede producir en forma de metástasis a distancia o por invasión directa de un tumor primario ${ }^{8}$. Si no diferenciamos la afectación secundaria de la metástasis, el principal tumor que origina neoplasias secundarias en la próstata es el vesical con un 57\% del total de tumores seguido del pulmonar con un $16 \%$, rectal $10 \%$ y pancreático 4\%. El más común de los tumores sólidos que origina metástasis en próstata es el escamoso bronquial (50\% de todas las metástasis), después el melanoma (27\%) y el resto es un miscelánea. En la serie de Bates con 51 casos de tumores secundarios prostáticos el $66,6 \%$ fueron originados por invasión directa frente al 33,3\% con afectación por metástasis a distancia. El 85\% de los casos de invasión directa se originaron por afectación de tumores vesicales y el $15 \%$ restante por adenocarcinomas de recto. Como vemos la afectación mas frecuente de neoplasia sólida secundaria de la próstata es la invasión directa de la misma por un tumor vesical, relegando a la afectación metastásica a ser un fenómeno excepcional $(0,69 \%$ de las neoplasias sólidas secundarias prostáticas) ${ }^{7}$.

Los linfomas y la leucemia son los tumores secundarios que más frecuentemente encontramos en la próstata (68\% vs $32 \%$ de otros tumores sólidos) si no diferenciamos entre sólidos y hematológicos. El linfoma prostático primario supone un 0,1\% de los linfomas diagnosticados y menos del 0,009\% de todas las neoplasias prostáticas. La afectación secundaria de la próstata por un linfoma sistémico es más frecuente9 ${ }^{9}$.

Es importante diferenciar el origen de la neoplasia secundaria de próstata diferenciando la metástasis de la invasión por contigüidad. La historia clínica y la exploración física son importantes a la hora de interpretar el origen de la neoplasia en el material de biopsia. El hecho de conocer un foco primario contiguo al órgano hace pensar en la posibilidad de invasión directa, mientras que una carcinomatosis generalizada o invasión sistémica por parte del primario hacen pensar en las metástasis en aparato genitourinario. La TAC o la RM pélvica pueden ser útiles para diferenciar estos diferentes orígenes sobretodo en el caso de los tumores de células transicionales. El PSA tiene un comportamiento poco constante, aunque se ha demostrado que los tumores primarios presentan inmunorreactividad para PSA con más frecuencia que los secundarios. Algunos casos raros de tumores primarios de próstata pueden mimetizar adenocarinomas metastásicos de otros órganos. Afortunadamente cuando son primarios son PSA Y PAP positivos lo cual los diferencia de los metastásicos. Otra diferencia es que la invasión exclusivamente vascular de la glándula es patrimonio de la afectación metastásica ${ }^{1}$.

Para concluir queremos resaltar que los pacientes con afectación tumoral secundaria de la próstata pueden presentar una serie de síntomas que son compatibles con la hiperplasia benigna de próstata ${ }^{10}$. La hematuria y el dolor pélvico son los sintamos más frecuentes que aparecen cuando hay afectación secundaria del tracto genitourinario. A pesar de que se trata de una entidad poco frecuente a la que rara vez se hace mención en la literatura médica, siempre debe quedar incluida en el diagnóstico diferencial de un paciente con esta sintomatología, sobre todo si tiene antecedentes de otra neoplasia ya conocida.

B I B L I O G R A F Í A

1. Bates AW, Baithun SI. The significance of secondary neoplasms of the urinary and male genital tract. Virchows Arch. 2002;440:640-7.

2. Epstein JI, Algaba F, Yang XJ. Tumours of the prostate. En: Eble JN, Sauter G, Epstein JI, Sesterhenn IA, editors. Tumours of the Urinary System and Male Genital Organs, Chapter 3. Lyon: IARC Press; 2004;160-208.

3. Zein TA, Huben R, Lane W, Pontes JE, Englander LS. Secondary tumours of the prostate. J Urol. 1955;133:615-6.

4. Klinger ME. Secondary tumors of the genito-urinary tract. J Urol. 1951;65:144-53.

5. Johnson DE, Chalbaud R, Ayala AG. Secondary tumours of the prostate. J Urol. 1974;112:507-8.

6. Goebbels R, Amberger L, Wernert N, Dhom G. Urothelial carcinoma of the prostate. Appl Pathol. 1985;3:242-54.

7. Bates AW, Baithun SI. Secondary solid neoplasms of the prostate:a clinico-pathological series of 51 cases. Virchows Arch. 2002;440:392-6.

8. Morichetti D, Mazzucchelli R, Lopez-Beltran A, Cheng L, Scarpelli M, Kirkali Z, et al. Secondary neoplasms of the urinary system and male genital organs. BJU Int. 2009;104: $770-6$.

9. Bell CR, Napier MP, Morgan RJ, et al. Primary non-Hodgkin's lymphoma of the prostate gland: case report and review of the literature. Clin Oncol (R Coll Radiol). 1995;7:409-10.

10. Zuazu JR, Iglesias R, Costa DR, Mayans AR, Brugarolas I, Roselló X, et al. Prostatic lymphoma and review of the literature. Actas Urol Esp. 2009;33:686-90. 
J. Barba Abad ${ }^{a, *}$, J.E. Robles García ${ }^{a}$, E. Tolosa Eizaguirre ${ }^{a}$, A. Panizo Santos ${ }^{b}$ y J.J. Zudaire Bergera ${ }^{a}$

à Departamento de Urología, Clínica Universidad de Navarra, Navarra, España bepartamento de Anatomía Patológica, Clínica Universidad de Navarra, Navarra, España

*Autor para correspondencia.

Correo electrónico: javiferbar@hotmail.com (J. Barba Abad).

\section{Perforación del colon ascendente tras litotricia extracorpórea por ondas de choque. Revisión de la literatura médica}

\section{Perforation of ascending colon after extracorporeal shock waves lithotripsy . A review of the literature}

\section{Sr. Director:}

La litotricia extracorpórea por ondas de choque (LEOC) desde su introducción en la década de 1980 representa un tratamiento eficaz y de primera línea en las litiasis renales y ureterales. Generalmente, las complicaciones surgidas tanto de forma inmediata como tardía suelen ser leves y controlables con tratamiento médico. En contadas ocasiones se presentan complicaciones mayores que requieren tratamiento quirúrgico.

Paciente de 34 años remitido desde su centro hospitalario de referencia con diagnóstico de litiasis de uréter lumbar derecho obstructiva para tratamiento con LEOC. Se realiza una primera sesión de tratamiento mediante LEOC con equipo Dornier Doly ${ }^{\circledR} 50$ generador electromagnético EMSE $220 \mathrm{~F}-\mathrm{XXP}{ }^{\circledR}$ en posición de decúbito prono, aplicando un total de 3.400 ondas de choque y 200 julios de energía $(58,82 \mathrm{~mJ} /$ onda), y permanece durante $2 \mathrm{~h}$ en observación tras finalizar el tratamiento, debiendo acudir un mes después a nuestra unidad para una revisión. El paciente regresa a su lugar de origen, acudiendo al servicio de urgencias de su hospital de referencia a las $24 \mathrm{~h}$ de la LEOC por presentar dolor abdominal agudo asociado a malestar general y febrícula. Tras realizársele pruebas analíticas y de imagen en dicho centro hospitalario, el servicio de cirugía decide realizar laparotomía exploradora, apreciando durante esta una perforación del colon ascendente, y llevando a cabo una hemicolectomía derecha y una anastomosis ileocólica. Tras un postoperatorio satisfactorio el paciente fue dado de alta de dicho centro.

La LEOC se considera una técnica con poca morbimortalidad y con el desarrollo de mejores sistemas para la localización del cálculo y el mayor control analgésico disminuyen las complicaciones de dicha terapéutica. No obstante, no resulta una técnica inocua y con relativa frecuencia se presentan complicaciones del tipo cólico renal (28\%), obstrucción ureteral (4\%), sepsis $(1,1 \%)$, deterioro de la función renal $(0,4 \%)$, hematomas renales $(0,4 \%)$ e hipertensión arterial, sin olvidar un porcentaje no despreciable de litiasis residual ${ }^{1}$. La mayoría de estas complicaciones y de otras menores, como dolor sobre la zona de impacto de la onda, se resuelven con tratamiento médico y actitud expectante.

Resulta extremadamente infrecuente la aparición de complicaciones mayores, tales como hematomas hepáticos, pancreatitis, arritmias cardiacas y perforaciones del tracto gastrointestinal.

Tras revisar exhaustivamente la literatura médica hemos encontrado 7 casos publicados de perforación del intestino delgado y 1 caso de perforación del colon sigmoide tras LEOC. En 1997 se publican los primeros 2 casos de perforación del intestino delgado secundaria a LEOC tras aplicar una media de 4.500 ondas de choque y realizar el tratamiento en decúbito prono, concluyendo que en determinados casos seleccionados según la localización ureteral y la posición del paciente es preciso reducir el número de ondas y el nivel de energía aplicado ${ }^{2,3}$. Kurtz et al presentan en 1999 un caso de perforación del intestino delgado posterior a litotricia extracorpórea tras colocar al paciente en decúbito prono ${ }^{4}$. En el año 2000 se documenta 1 caso de perforación del intestino delgado pos-LEOC (4.000 ondas de choque) que precisó resección ileal de $6 \mathrm{~cm}$ y posterior anastomosis terminoterminal, implicando la posición de decúbito prono y altos niveles de energía como posibles factores de riesgo para dicha complicación ${ }^{5}$. En ese mismo año Lipay et al presentan el caso de un paciente de 32 años con perforación del colon sigmoide secundaria a LEOC tras aplicar 3.000 ondas de choque a $7 \mathrm{kV}$, requiriendo resección del segmento afecto y posterior anastomosis colorrectal ${ }^{6}$. En 2001 se publican 2 nuevos casos de perforación intestinal tras LEOC en decúbito prono, presentando uno de los pacientes dos perforaciones de aproximadamente $2 \mathrm{~mm}$ en yeyuno, indicando que tanto los antecedentes quirúrgicos abdominales como la posición en prono deben ser tenidas en cuenta para realizar la litotricia 